\title{
Homonymous hemianopsia versus unilateral spatial neglect rehabilitation strategies
} in stroke patients

\author{
Adriana Elena Bulboaca ${ }^{1,2}$, Angelo Bulboaca ${ }^{1,2}$, Ioana Stanescu ${ }^{1,2}$, Paul-Mihai Boarescu ${ }^{1}$, Ioana Chirilă ${ }^{3}$, \\ Alexandra Bulboaca ${ }^{1}$, Gabriela Dogaru ${ }^{1,2}$
}

Corresponding author: Paul-Mihai Boarescu ${ }^{1}$, E-mail: paul.boarescu@gmail.com

p: $67-73$

\section{1. "Iuliu-Hatieganu" University of Medicine and Pharmacy, Cluj-Napoca, Romania \\ 2. Clinical Rehabilitation Hospital, Cluj-Napoca, Romania \\ 3. County Clinical Emergency Hospital, Cluj-Napoca, Romania}

\begin{abstract}
Visual rehabilitation therapy is one of the most problematic issue in stroke rehabilitation. The difficulties consist in specific assessment of visual deficit and poor results reports by the authors of the clinical studies. Opposite, experimental studies reports encouraging results that give hopes in this specific rehabilitation therapy. There are still difficult to analyze different aquisitions concerning various visual residual deficits after stroke, the main rehabilitation targgets being motor rehabilitation in order to ensure at least a partial autonomy in day by day life. All the studies that proved there are chances for a better quality of life if there is an improvement of visual abilities together with motor and cognitive skills with a better rehabilitation prognosis. The aim of this paper is to make a brief report regarding two of the most important visual deficits after stroke as are homonymous hemianopsia and neglect. Starting with differential diagnosis, neuroplasticity and specific rehabilitation available method, the main issues are discussed. a better understanding of phenomena that are associated with spontaneous rehabilitation, or enhancing the progress of recuperation by various method, could be able to bring a new light and hopefully better results in rehabilitation for these patients
\end{abstract}

Key words: stroke, visual impairment, visual rehabilitation, neuroplasticity, homonymous hemianopsia,

\section{Introduction}

Homonymous hemianopsia $(H H)$ is one of the most common disorders following ischemic stroke, usually associated with post-chiasmatic lesions, which has serious impact on motor rehabilitation, visual skills, driving, reading, and self-care activities (1). Transitive hemianopsia that are followed by spontaneous recovery can be associated with high levels of glycemia or hypertension and with other neurological conditions as are transient ischemic attack, migraine, occipital, parietal or temporal seizures, hemorrhagic stroke, brain tumors or trauma, demyelination process, antiphospholipidic syndrome, central nervous system infections or Alzheimer's disease (2-7). An important step in recognising hemianopsia is the differential diagnosis with hemineglect disorders that can both followed after stroke onset. For this differential diagnosis there is needed to take in consideration some important elements as are: subjective complaint of the patients, aetiology and localisation of the lesion, extension of the visual deficit by clinical examination, specific visual field testing techniques for diagnosis of visual blindness (scotoma) or other types of visual field defects (8). The perimetry results depends on patient attention and understanding the tasks, fixation maintaining ability, and the examination accuracy. automated perimetry, most used in clinical practice can offer important information useful for differential diagnosis (9). Improvement of $\mathrm{HH}$ after stroke can constitute a serious challenge with an impact on entire rehabilitation strategies and results, especially in ischemic stroke patients. Despite the fact of high reported prevalence of visual disabilities associated with stroke (approximately one third of patients present visual disorders after stroke), the visual rehabilitation therapy is inconsistent and has no precise milestones that can constitute a real protocol in stroke rehabilitation therapies schema (10). Moreover, visual field deficits is relatively undervalued in the clinical setting due to poor prognosis and imprecise results after different rehabilitation methods (11). HH persistence is a negative predictor for patients outcome despite the motor and speech rehabilitation therapies efforts (12). It is possible that adding a systematic vision rehabilitation to other methods for stroke rehabilitation could consistently improve patient's quality of life $(13,14)$.

Many strategies that already developed in this research field of $\mathrm{HH}$ rehabilitation met scepticism in 
scientifically communities, therefore, clinical approaching was not constant followed, and there is no consensus regarding the methods and therapies applied in this form of visual disabilities. There are also only few studies regarding different methods applied for $\mathrm{HH}$ rehabilitation with various approaching that have a common technique of repetitive exercises of a special visual task that can hopefully stimulate the visual spared abilities in order to reach a performance that can be useful in improving day by day activities (12). Unfortunately, during these therapies, patients expectations are high, and they are followed by a decline after their hopes are not accomplished $(15,16)$. Despite of these concepts there are reports that showed that visual field defects can be partially reparable and certain degrees of deficiencies can be improved $(17,18)$. Improving $\mathrm{HH}$ together with motor skill, based on various strategies, can constitute a serious background for developing more detailed rehabilitation strategies that could lead to and partially or even total indepented life in stroke patients $(15,19)$. Patient who suffered an acquired visual disability as is hemianopsia have to make dramatic behavioural and compensatory adjustments in order to trying to keep their independence in a world strongly relied on visual stimuli. Understanding the function of the visual system in and neuroplasticity phenomena can be an important step toward a better rehabilitation of these patients, improving their outcome chances, and finally their life quality.

Unilateral spatial neglect (USN) consist in unresponsiveness to any visual or tactile stimulus on one side of the body (contralateral side defect), that cannot be attributed to sensory or motor deficit (20). This condition is associated with lesions in the right hemisphere, particularly in the right hemisphere (inferior parietal lobe, superior temporal gyrus and/or inferior frontal gyrus) and with poor prognosis (21). There was reported that approximately $85 \%$ of the patients with subacute or acute ischemic lesions associated with stroke, presents variable grades of USN, and there is no universal agreement concerning the assessment methods for USN evaluation (22). Topographical evaluation of the lesions showed that neglect was more common and severe in posterior associated cortical lesions, and behavioural assessment as is automatic right side orientation was the most sensitive clinical estimation of USN (22). Clinical presentation of the patient is leading to a confusion with $\mathrm{HH}$ at the first evaluation of the patient, but a detailed observation of the patient concerning spatial orientation is concluding to a correct diagnosis. The automatic tendency orientation toward the right side of their environmental space, in the presence of a visual stimuli constitute a strong indicator of USN. The role of gaze direction in USN seems to be a particular mechanism used by the patient in order to adapt to USN (23). The gaze can be processed despite the fact the patient is unable to attend to it, being attributable to residual abilities in sensorial processing function that can modulate attention toward the left side of the environmental space (24). Despite the fact the initial evaluation can be interpreted as an artefact associated with clinical presentation of the patient due to ischemic lesions, a careful clinical and imagistic evaluation may conclude to a correct diagnosis of USN. The grade of spatial perception can be also variable, according with the possibility to partially compensate the defect by residual functions. If the damage produced by ischemia is large and a cystic region is forming in the place of ischemic tissue (where the neuronal death was produced) as the consequence of stroke, the rehabilitation process is expected to be very poor (25). The aim of this paper is to emphasize the main rehabilitation techniques in $\mathrm{HH}$ and USN rehabilitation, based on physiological support of visual system in brain circuitries, on plasticity and reorganisation proprieties of neuronal networks and on individual patients abilities to improve their conditions, according with cognition, age and stroke features.

\section{Ethiopathogenesis and functional impact}

The main ethiopatogenetic factors associated with $\mathrm{HH}$ are represented by ischemic stroke, mainly associated with brain lesions in posteriors cerebral artery territories and middle cerebral artery territories (26), but can be also associated with traumatic brain injury or brain tumors and cerebral infectious disorders (27). Moreover, the visual field defect it is associated with motor, sensory, cognitive deficits and/or aphasia or depression. All of these disabilities contribute alone and together to a serious impairment of life quality, constituting an vicious circle, that consist in aggravation one each other, and being, in this way, difficult to improve. After an ischemic stroke, motor deficit can be serious negatively influenced by visual disabilities (Barthel index that measure the performance in activities of daily living decreasing when visual disabilities are associated), 
quality of life being more affected in young patients than in older ones (28). High dependency in day by day activities and depression that are persistent at 3 years after stroke onset, are factor that have a major contribution to a low quality of life in stroke survivors (29). The mood and emotional disturbances may be treated or prevented by improving of rehabilitation progress (regarding motor/sensorial/visual disabilities) in a period of 1-2 years after stroke onset decreasing the stressor factors in stroke survivors and essentially contributing to the quality of life (30). Neglect it is mainly a consequence of disrupted internalized representation of the environment perception with associated cortical and white-matter tracts dysfunction having as a consequence an interhemispheric lack of communication and manifested as neglect in clinical assessment (most used test for clinical assessment test are Catherine Bergego scale, line bisection test and star cancellation test) (31).

\section{Neuronal plasticity and reorganisation of neuronal networks}

In general, spontaneous improvement of $\mathrm{HH}$ occurs in about $50 \%$ of stroke survivors, in the first $1-3$ month after injury (32). After 6 months spontaneous recovery need to be interpreted with prudence because significantly improvement is improbable (32). Spontaneous recovery is based on a cascade of event that follow after ischemia onset and consists in molecular, cellular and electrophysiological changes which will enhance neuronal recovery. All of these phenomena are triggering cortical reorganisation and regeneration being the background of functional neuronal recovery. Experimental studies showed that these events begin very early, in few hours after stroke onset, reaching a peak in 7-14 days and being nearly complete in approximately 30 days. Evidence from animal studies shows that after ischemic injury, a cascade of genetic, molecular, cellular, and electrophysiological events is triggered which promote neural recovery. Together, these events drive cortical reorganization and regeneration, and provide the neural substrate for spontaneous recovery. In rodent models, these events begin within hours after stroke, peak at 7-14 days, and are nearly complete at 30 days (33). Recovery continue in the next month but more slowly, therefore became important to accelerate and improve recovery in the first 30 days after stroke $(34,35)$. There are various difficulties in clinical practice to realize this goal due to instability of some patients in the first 14 days after stroke. In the meaning time there are reports that sustain that active rehabilitation in the first $24 \mathrm{~h}$ is dangerous and can be harmful (36). One of the mechanism associated to recovery after stroke consists in axonal sprouting in cortical areas adjacent to infarct being detectable with anatomical mapping of cortical circuits (demonstrated by immunohistochemical staining patterns of axonal proteins, such as neurofilaments or synapse-associated proteins) one month after the stroke but the signal that initiates axonal sprouting is not known $(37,38)$. After the recovery reach the point where from the acquisitions are very slow, the patients can concomitant develop a deformed perception of the visual field (spherical deformation of the objects at the edge of the blind visual field). The hypothesis enhanced was that cortical reorganization can occur in the human adult visual system after loss of cortical input as a consequence of cortical reorganization (39). There are several reports that sustain that the V1 area has the possibility to exert plasticity phenomena and thus to recuperate, at least partially, functionality $(40,41)$. The same process is taking place in old patients that present an age related macular degeneration develop a large scale reorganisation of visual cortex after deafferentation due to the lack of visual stimuli that can arrive at visual cortex and (42). There is unreported evolution of the patients where the homonymous hemyanopsia is superimposed on a retina with age related macular degeneration. Anyhow, multiple causes that can affect the visual perception (ophthalmological causes and neurological causes), when are present in the same patient, can decrease considerable the rehabilitation outcome and can constitute together a poor prognosis for targeting an independent day by day life. Stroke recovery is based on the new system of neuronal connections or on the functional properties of neurons in a recovering area (43). Regarding the USN rehabilitation, the functional outcome depends on neglect type: personal, peripersonal and extrapersonal. Spaccavento at all reported that there are very variable rehabilitation grades of patients with neglect (that can have differed in motor and cognitive deficits at admission and discharge) according with the patient's age and lesion extension (44).

\section{Methods for homonymous hemianopsia rehabilitation}

Current knowledge offer four main strategies for $\mathrm{HH}$ rehabilitation: replacing partially the damaged visual field with intact visual field by prisms (optical therapy), partially restoring the lost visual field 
(restorative therapy), stimulating detection capacities in blind visual field (stimulation therapy) and reorganisation of visual function by eye movements in order to enlarge the cortical regions for visual perception (compensatory therapy) $(12,45)$.

1. Optical therapy - consists in applying optical devices that can deviate the blind visual field toward the functional intact visual field, offering to the patient a correction with prismatic lenses. A high power prismatic lens (30-40 dioptre) is orientated towards the hemianoptic field, at about the level of the limbum (46). By peripheral location of the prism will lead to peripheral exotropia that will put the object from blind field into the normal functional field, enlarging the degrees of perception in blind field with about 20 degrees (47). Lower dioptre (D) prismatic lenses as are those below $20 \mathrm{D}$ can only provide about 10 degrees of enlargement for blind visual field (48). The long term success rate of optic therapy applying, with prismatic lenses correction, is very variable as is reported by Bowers at all (27 $81 \%$ ) from very low to good compliance (47). The main reasons for low compliance were the anxiety of the patients due to sudden visibility of some objects in the visual field, and consequently, confusion and anxiety (47). Other objections that decrease the compliance are difficulties to reading with this optical correction (very often due to necessity of bifocal correction), difficulties to step on descending stairs (may produce diplopia) (47). There is also important to take in consideration the costs for Fresnel prisms that are used as a permanent correction in optical therapy for homonymous hemianopsia (49). There are also patients that can have a good toleration of prismatic lens correction that means that are already used to wear and they don't need to change the head position and posture of the body, in order to get a better image of the environmental visual field. There is a study that reported that approximately $15-17 \%$ of the patients do not meet the criteria for prismatic lens correction, due to low tolerance (withdrew in the first week) or due to the reason they were not eligible; the rest of participants in Bowers at al study confirmed that prism correction helped them to avoid obstacles during mobilisation (47). Monoocular prism correction should be taken in consideration for the patients who present age related macular degeneration in an advanced stage (50). Based on acceptance of these devices (Fresnel prism) optical correction of visual field defect can constitute a modality to reduce the patients visual discomfort during the rehabilitation process. There are needed more studies to prove the reliability and efficacy of prismatic correction for these patients due to multiple disabilities that they presenting at the time of the admission into the hospital, that can constitute a serious burden from physical and psychological point of view. Anyhow, this treatment can bring a hope for improving patient condition during the rehabilitation therapy, being one of the reachable improvement of functionality in stroke patients.

2. Restorative therapy - is based also on the brain plasticity and on its ability for reorganisation after an injury. Training the patient to perceive the light at the limits between blind visual field and healthy visual field an enlargement of 5 degrees can be obtained (8). The stimulation of the border zone between blind visual field and healthy visual field can lead to neuronal plasticity and neuronal network reorganisation but the proves in this direction are inconsistent. The difficulties to objectify this theory are based on different devices for visual field assessment in clinical trials and inconsistent results (12). Therefore visual restoration therapy remains controversial and the scientific community has a high grade of scepticism regarding its results.

3. Stimulation therapy - consists in stimulation of existing visual capacities in blind visual field by training the patients to detect visual stimuli targeted on the spared area in the blind visual field. The stimulus consists usually in a flickering light that has to be detected by the patients. After one year of intensive training some authors reported the ability of the patients to identify the lightening stimuli and, in the same time, an expansion of visual field of 20 degrees (46). There are sceptic approaching of these results due to lack of indication of the topography of the lesion during these reports, and due to lack of evidences of brain plasticity that can support the findings. More studies are needed to transpose the animal studies that proved brain plasticity due to visual rehabilitation process to the human subjects, therefore at the moment are interpreted as speculative $(51,12)$.

4.Compensatory therapy - saccades (alone or with auditory stimulus together) - consists in training the patients daily during a long period of time. The method consists in training the patient's to perform saccades in the blind field, in order to enlarge the area of perception, together with enhancing the spatial organisation of ocular movement toward the hemianoptic field, by increasing the number of 
fixations toward the visual field (usually the method consist in stimulation of flashing stimuli vision) (12). The basis of this visual achievement is changing the brain activity by activation of striate and extra-striate cortex and increasing their performances (12).

\section{Methods for unilateral spatial neglect rehabilitation}

The interest for unilateral spatial neglect rehabilitation is specially directed toward rehabilitation of left spatial neglect after ischemic stroke in right hemisphere, which is more common and more often associated with left hemisphere stroke (52). The techniques are variable and consists mainly in different approaching. One of the technique is based on the stimulation of gaze direction toward left using top-down stimuli that are variable in intensity, number or duration for about 20 hours of training due to 20 days (53). Other rehabilitation methods are using ascending sensory stimulations as are vestibular stimulations, optokinetic stimulations and electrical stimulation of the neck muscles with good results (54). A new and promising methods for unilateral spatial neglect rehabilitation consist in transcranial magnetic stimulation and transcranial direct current stimulation that are non invasive technique with encouraging results (55). The fundamental theory that is the basis of these method consist in creating a competition between left and right hemisphere with enhancing the activity of right hemisphere in order to reduce the unilateral spatial neglect in right hemisphere lesions (56).

\section{Future perspectives}

Despite the fact the methods regarding homonymous hemianopsia and unilateral spatial neglect rehabilitation are different, they have one common concept as is stimulation of cortical residual functions for new perception in order to reduce the blind deficit in visual field. This concept is based on neuroplasticity of neuronal network that prove the learning process of the brain can be continued in adulthood and can constitute a starting point for various abilities rehabilitation that disappeared after lesions. There are needed more clinical studies in order to organize the methods proved to be efficient in this patients in order to establish a clinical valuable protocol. Novel techniques as are transcranial magnetic stimulation and transcranial direct current stimulation could bring a new hope for the patients who suffer from these deficits that could impair seriously their entire rehabilitation progress.

\section{Conclusions}

Further development of these strategies, based on experimental and clinical studies are needed. Interdisciplinary team that is specialized on different types of training for patient rehabilitation needs to collaborate for the best results. All the data reported are promising and their implementation and future development can significantly improve the quality of life for stroke patients survivors.

\section{References}

1. Loetscher $\mathrm{T}$, Chen $\mathrm{C}$, Wignall $\mathrm{S}$, Bulling A, Hoppe S, Churches O, et al. A study on the natural history of scanning behaviour in patients with visual field defects after stroke. BMC neurology. 2015 Dec;15(1):64.

2. Strowd RE, Wabnitz A, Balakrishnan N, Craig J, Tegeler $\mathrm{CH}$. Clinical Reasoning: Acute-onset homonymous hemianopia with hyperglycemia: Seeing is believing. Neurology. 2014 Apr 15;82(15):e129-33.

3. Hentschke MR, Sussela AO, Marrone LC, Costa BE, Poli-de-Figueiredo CE， Gadonski G. Reversible hemianopsia in postpartum due to posterior reversible encephalopathy syndrome in pregnant with late eclampsia. J Bras Nefrol. 2016 Jun;38(2):265-8.

4. Dwivedi RA, Dwivedi RE, Durnian JM, Young CA. Tumefactive demyelination: an unusual cause of a spontaneously resolving homonymous hemianopia. BMJ case reports. 2013 Jun 21;2013:bcr2013009363.

5. Ho DK, Ramessur R, Gupta M, Mathews JP. Homonymous hemianopia in the primary antiphospholipid syndrome. BMJ case reports. 2017 Feb 8;2017:bcr2016218660.

6. Yazici B, Kivanc SA. Isolated bitemporal hemianopsia due to traumatic chiasmal syndrome. Ulus Travma Ve Acil Cerrahi Derg. 2016 Jan 1;22(1):97-9.

7. Ishiwata A, Kimura K. Homonymous hemianopsia associated with probable Alzheimer's disease. J Nippon Med Sch. 2016 Apr 15;83(2):87-92.

8. Kerkhoff G. Neurovisual rehabilitation: recent developments and future directions. Journal of Neurology, Neurosurgery \& Psychiatry. 2000 Jun 1;68(6):691-706.

9. Phu J, Khuu SK, Yapp M, Assaad N, Hennessy MP, Kalloniatis M. The value of visual field testing in the era of advanced imaging: clinical 
and psychophysical perspectives. Clin Exp Optom. 2017 Jul;100(4):313-32.

10. Raz N, Levin N. Neuro-visual rehabilitation. J Neurol. 2017 Jun 1;264(6):1051-8.

11. Plow EB, Maguire S, Obretenova S, PascualLeone A, Merabet LB. Approaches to rehabilitation for visual field defects following brain lesions. Expert Rev Med Devices. 2009 May 1;6(3):291-305.

12. Pouget MC, Lévy-Bencheton $\mathrm{D}$, Prost $\mathrm{M}$, Tilikete C, Husain M, Jacquin-Courtois S. Acquired visual field defects rehabilitation: critical review and perspectives. Annals of physical and rehabilitation medicine. 2012 Feb 1;55(1):53-74.

13. Bulboaca A, Bulboaca A, Stanescu I, Blidaru M, Bulboaca AI, Nicula C, Dogaru G. Post-stroke visual rehabilitation-the impact on life quality of the stroke survival patients-a brief review. Balneo Research Journal. 2019 Feb 1;10(1):17-23.

14. Dogaru G, Ispas A, Bulboacă A, Motricală M, Stănescu I. Influence of balnear therapy at Băile Tuşnad on quality of life of post-stroke patients. Balneo Research Journal. 2017;8(4):201-205

15. Bulboacă AE, Bolboacă SD, Bulboacă AC. Ethical considerations in providing an upper limb exoskeleton device for stroke patients. Medi hypotheses. 2017 Apr 1;101:61-4.

16. Rocksmith ER, Reding MJ. New developments in stroke rehabilitation. Curr Atheroscler Rep. 2002 Jul;4(4):277-84.

17. Geuzebroek AC, van den Berg AV. Impaired visual competition in patients with homonymous visual field defects. Neuropsychologia. 2017 Mar 1;97:152-62.

18. Sabel BA, Trauzettel-Klosinksi S. Improving vision in a patient with homonymous hemianopia. J Neuroophthalmol. 2005 Jun;25(2):143-9.

19. Dogaru G, Marieta M, Bulboacă A, Ciumărnean L, Stănescu I. The effect of carbonated mineral water and mofette treatment in Baile Tusnad after ischemic stroke-a case report. Balneo Research Journal. 2018 Jan 1;9(1):05-10.

20. Luvizutto GJ, Rizzati GR, Fogaroli MO, Rodrigues RT, Ribeiro PW, de Carvalho Nunes HR,et al. Treatment of unilateral spatial neglect after stroke using transcranial direct current stimulation (ELETRON trial): study protocol for a randomized controlled trial. Trials. 2016 Dec;17(1):479.
21. Denes G, Semenza C, Stoppa E, Lis A. Unilateral spatial neglect and recovery from hemiplegia: a follow-up study. Brain. 1982 Sep 1;105(3):543-52.

22. Azouvi P, Jacquin-Courtois S, Luauté J. Rehabilitation of unilateral neglect: Evidencebased medicine. Ann Phys Rehabil Med. 2017 Jun;60(3):191-197.

23. Maravita A, Posteraro L, Husain M, Vuilleumier P, Schwartz S, Driver J. Looking at human eyes affects contralesional stimulus processing after right hemispheric stroke. Neurology. 2007 Oct 16;69(16):1619-21.

24. Radoeva PD, Prasad S, Brainard DH, Aguirre GK. Neural activity within area V1 reflects unconscious visual performance in a case of blindsight. J Cogn Neurosci. 2008 Nov;20(11):1927-39.

25. Stoerig P, Zontanou AH, Cowey A. Aware or unaware: Assessment of cortical blindness in four men and a monkey. Cerebral Cortex. 2002; 12:565-574.

26. Sabel BA, Kruse R, Wolf F, Guenther T. Local topographic influences on vision restoration hot spots after brain damage. Restorative neurology and neuroscience. 2013 Jan 1;31(6):787-803.

27. Sabel BA, Flammer J, Merabet LB. Residual vision activation and the brain-eye-vascular triad: Dysregulation, plasticity and restoration in low vision and blindness-a review. Restorative neurology and neuroscience. Restor Neurol Neurosci. 2018;36(6):767-791.

28. Kim JS, Choi-Kwon S, Kwon SU, Lee HJ, Park KA, Seo YS. Factors affecting the quality of life after ischemic stroke: young versus old patients. J Clin Neurol. 2005 Apr;1(1):59-68.

29. Choi-Kwon S, Choi JM, Kwon SU, Kang DW, Kim JS. Factors that Affect the Quality of Life at 3 Years Post-Stroke. J Clin Neurol. 2006 Mar;2(1):34-41.

30. Kim JS. Post-stroke Mood and Emotional Disturbances: Pharmacological Therapy Based on Mechanisms. J Stroke. 2016 Sep;18(3):244-255.

31. Ting DS, Pollock A, Dutton GN, Doubal FN, Ting DS, Thompson M, et al. Visual neglect following stroke: current concepts and future focus. Surv Ophthalmol. 2011 MarApr;56(2):114-34.

32. Zhang WN, Pan YH, Wang XY, Zhao Y. A prospective study of the incidence and correlated factors of post-stroke depression in China. PLoS One. 2013 Nov 18;8(11):e78981. 
33. Krakauer JW, Carmichael ST, Corbett D, Wittenberg GF. Getting neurorehabilitation right — what can we learn from animal models? Neurorehabil Neural Repair. 2012;26(8):923-31.

34. Coleman ER, Moudgal R, Lang K, Hyacinth HI, Awosika OO, Kissela BM, et al. Early Rehabilitation After Stroke: a Narrative Review. Curr Atheroscler Rep. 2017 Nov 7;19(12):59.

35. Moon SK, Alaverdashvili M, Cross AR, Whishaw IQ. Both compensation and recovery of skilled reaching following small photothrombotic stroke to motor cortex in the rat. Exp Neurol. 2009;218(1):145-53.

36. Stinear C, Ackerley S, Byblow W. Rehabilitation is initiated early after stroke, but most motor rehabilitation trials are not: a systematic review. Stroke. 2013;44(7):2039-45.

37. Li S, Nie EH, Yin Y, Benowitz LI, Tung S, Vinters HV et al. GDF10 is a signal for axonal sprouting and functional recovery after stroke. Nat Neurosci. 2015 Dec;18(12):1737-45.

38. Clarkson AN, López-Valdés HE, Overman JJ, Charles AC, Brennan KC, Thomas Carmichael S. Multimodal examination of structural and functional remapping in the mouse photothrombotic stroke model. J Cereb Blood Flow Metab. 2013 May;33(5):716-23.

39. Dilks DD, Serences JT, Rosenau BJ, Yantis S, McCloskey M. Human adult cortical reorganization and consequent visual distortion. J Neurosci. 2007 Sep 5;27(36):9585-94.

40. Kapadia MK, Gilbert CD, Westheimer G. A quantitative measure for short-term cortical plasticity in human vision. J Neurosci. 1994 Jan;14(1):451-7.

41. Kalarickal GJ, Marshall JA. Rearrangement of receptive field topography after intracortical and peripheral stimulation: the role of plasticity in inhibitory pathways. Network. 2002 Feb;13(1):1-40.

42. Baker CI, Peli E, Knouf N, Kanwisher NG. Reorganization of visual processing in macular degeneration. J Neurosci. 2005;25:614-618.

43. Carmichael ST, Kathirvelu B, Schweppe CA, Nie EH. Molecular, cellular and functional events in axonal sprouting after stroke. Exp Neurol. 2017 Jan 1;287:384-94.

44. Spaccavento S, Cellamare F, Falcone R, Loverre A, Nardulli R. Effect of subtypes of neglect on functional outcome in stroke patients. Ann Phys Rehabil Med. 2017 Nov;60(6):376-381.
45. Gao Y, Sabel BA. Microsaccade dysfunction and adaptation in hemianopia after stroke. Restor Neurol Neurosci. 2017;35(4):365-376.

46. Peli E. Field expansion for homonymous hemianopia by optically induced peripheral exotropia. Optom Vis Sci. 2000 Sep;77(9):453-64.

47. Bowers AR, Keeney K, Peli E. Communitybased trial of a peripheral prism visual field expansion device for hemianopia. Arch Ophthalmol. 2008 May;126(5):657-64.

48. Szlyk JP, Seiple W, Stelmack J, McMahon T. Use of prisms for navigation and driving in hemianopic patients. Ophthalmic Physiol Opt. 2005;25:128-135.

49. Perlin RR, Dziadul J. Fresnel prisms for field enhancement of patients with constricted or hemianopic visual fields. J Am Optom Assoc. 1991 Jan;62(1):58-64.

50. Parodi MB, Toto L, Mastropasqua L, Depollo M, Ravalico G. Prismatic correction in patients affected by age-related macular degeneration. Clin Rehabil. 2004 Nov;18(7):828-32.

51. Khan S, Leung E, Jay WM. Stroke and visual rehabilitation. Top Stroke Rehabil. 2008 JanFeb;15(1):27-36.

52. Beis JM, Keller C, Morin N, Bartolomeo P, Bernati T, Chokron S, et al. Right spatial neglect after left hemisphere stroke: qualitative and quantitative study. Neurology. 2004 Nov 9;63(9):1600-5.

53. Smania N, Bazoli F, Piva D, Guidetti G. Visuomotor imagery and rehabilitation of neglect. Arch Phys Med Rehabil. 1997 Apr 1;78(4):430-6.

54. Pérennou DA, Leblond C, Amblard B, Micallef JP, Hérisson C, Pélissier JY. Transcutaneous electric nerve stimulation reduces neglect-related postural instability after stroke. Arch Phys Med Rehabil. 2001 Apr 1;82(4):440-8.

55. Miniussi C, Cappa SF, Cohen LG, Floel A, Fregni F, Nitsche MA, et al. Efficacy of repetitive transcranial magnetic stimulation/transcranial direct current stimulation in cognitive neurorehabilitation. Brain Stimul. 2008 Oct;1(4):326-36.

56. Jacquin-Courtois S. Hemi-spatial neglect rehabilitation using non-invasive brain stimulation: or how to modulate the disconnection syndrome?. Arch Phys Med Rehabil. 2015 Sep 1;58(4):251-8. 